\title{
Carbon Nanotubes in Cancer Research and Therapy
}

\author{
César A C Sequeira* \\ Centro de Física e Engenharia de Materiais Avançados (CeFEMA), Instituto Superior Técnico (IST), Universidade de Lisboa, Av.Rovisco \\ Pais, 1, 1049-001 Lisboa, Portugal \\ *Corresponding author: César A C Sequeira, Centro de Física e Engenharia de Materiais Avançados (CeFEMA), Instituto Superior \\ Técnico (IST), Universidade de Lisboa, Av.Rovisco Pais, 1, 1049-001 Lisboa, Portugal
}

\begin{tabular}{|c|c|}
\hline ARTICLE INFO & ABSTRACT \\
\hline Received: 㓞 February 10, 2020 & The author envisions carbon nanotube-based molecular nanotechnology's role \\
\hline Published: February 19, 2020 & $\begin{array}{l}\text { in developing advanced multifunctional materials and molecular-scale electronic and } \\
\text { computing devices, sensors, actuators and machines. Then, he briefly reviews and }\end{array}$ \\
\hline
\end{tabular}

Citation: César A C Sequeira. Carbon

Keywords: Carbon nanotubes; Fullerenes; Cancer therapy; Drug delivery; Functionalization

Biomed J Sci \& Tech Res 25(5)-2020. BJSTR.

MS.ID.004252.

\section{Mini Review}

During the second half of the 20th century, a microelectronics revolution moved us from vacuum tube electronic computers to silicon based integrated electronic devices. As the 21st century began this revolution of scaling, which has traditionally followed Moore's law, is facing major obstacles. To move forward, the science and technology community has tentatively moved from microdevice technology to the emerging field widely known as nanotechnology. The science and technology of nanoscale materials, devices, and applications in areas such as computers, sensors, actuators, and machines fall within the realm of nanotechnology. For the purposes of this article, we consider atoms and molecules (or extended atomic or molecular structures) to be the basic units or building blocks of fabricating future generations of electronics, materials, devices, and applications. At nanometer-length scales, many diverse fields and their associated technologies start to merge because the material properties are derived from the molecular building blocks.

The molecularly perfect structure that produces exceptionally strong structural and mechanical behavior in one class of system applications can also produce the exotic electronic and chemical behavior in another class.

The role of modern computational techniques has become critically important in nanotechnology development. The length and time scales of nanoscale systems and phenomenon have shrunk to where we can directly address them with computer simulations and theoretical modeling with high accuracy. The rapidly increasing computing power used for large-scale and high-fidelity simulations make it increasingly possible for nanoscale simulations to be also predictive. Thus, modern nanotechnology is emerging as a fundamental engineering analysis tool for novel nanodevice design in the way that continuum finite-element analysis has been used for designing and analyzing most engineering systems.

This article's main objective is to introduce the underlying molecular-scale building blocks fullerenes and carbon nanotubebased molecular materials in all four basic core areas (functional or smart materials, nanoscale electronics, molecular-scale sensors and actuators, and molecular machines or motors with synthetic materials) of nanotechnology which create the many applications possible in the future in vastly different areas. Here, carbon nanotubes, CNTs, and fullerenes as building blocks are described, as well as some of their physical, chemical, mechanical and electronic properties, which render them useful for cancer therapy, and other domains of pharmaceutical and medical research.

\section{Nanotubes and Fullerenes}

Fullerenes are close-caged molecules containing only hexagonal and pentagonal interatomic bonding networks. Nanotubes are large linear fullerenes with aspect ratios as large as 1000 to 100000 . 
Since their discovery about 30 years ago [1], researchers have extensively investigated carbon nanotubes (and many derivatives of fullerenes, such as nano cones, nano springs, and nanotrusses) theoretically and experimentally. Although many nanoscale fullerene materials (which consist entirely of carbon atoms) occur regularly in experiments, controlled production of many fullerenes and nanotubes with well-defined characteristics has not occurred. A single-wall carbon nanotube (SWNT) is basically a rolled-up shell of graphene sheet made of benzene-type hexagonal carbon rings [1]. Half-fullerenes cap the shell's ends. Unlike graphene, fullerenes consist of a combination of hexagons and pentagons to satisfy the Euler rule that governs the number of pentagons in any closed-cage structure. Multiwall nanotubes (MWNT's) are more common and can be produced in bulk with current experimental techniques.

An MWNT is a rolled-up stack of graphene sheets in concentric SWNT's, again with the ends capped by half-fullerenes. The nomenclature $(n, m)$ used to identify each SWNT refers to integer indices of two graphene unit lattice vectors corresponding to a nanotube's wrapping index, known as the chiral vector. Chiral vectors determine the directions along which the graphene sheets are rolled to form shell structures and are perpendicular to the tube axis vectors [1]. Nanotubes of type $(n, n)$, are commonly called Armchair Nanotubes because of their $\backslash_{-} / \backslash_{-} /$shape perpendicular to the tube axis. They have a symmetry along the tube axis with a short unit cell $(0.25 \mathrm{~nm})$ that can be repeated to make the entire section of a long nanotube. Nanotubes of type $(n, 0)$ are called Zigzag Nanotubes because of their $\bigwedge \bigwedge$ shape -- they have a short unit cell $(0.43 \mathrm{~nm})$ along the tube axis. All remaining nanotubes are called Chiral Nanotubes and have longer unit cell sizes along the tube axis. Details of the symmetry properties of the nanotubes and how to roll a sheet to make nanotubes of different chirality's appear elsewhere [1].

SWNT's and MWNT's are interesting nanoscale materials for these reasons:

- An SWNT can be either metallic or semiconducting, depending on its chiral vector $(n, m)$. The rule is that when the difference $n-m$ is a multiple of three, a metallic nanotube is obtained. If the difference is not a multiple of three, a semiconducting nanotube is obtained. We can connect nanotubes with different chirality's to create nanotube heterojunctions; these junctions form a variety of nanoscale molecular electronic device components.

- $\quad$ SWNT's and MWNT's have good electromechanically properties because the $2 \mathrm{D}$ arrangement of carbon atoms in a graphene lattice allows large out-of-plane distortions, while the strength of carbon-carbon in-plane bonds keeps the graphene sheet exceptionally strong against any in-plane fracture or distortion. All distortions induced in a simulation or observed in a static snapshot of an experiment appear to indicate high elasticity of the nanotubes and point toward their possible use as a lightweight, highly elastic, strong fibrous material.
- Because nanotubes are hollow, tubular, caged molecules, they can act as lightweight, large-surface-area packing material for gas storage and hydrocarbon fuel storage devices, as well as nanoscale containers for molecular drug delivery and casting structures for making nanowires and nanocapsulates.

These three qualities suggest a variety of possible applications. The nanotube heterojunctions with electronic switching properties could influence the next generation of computer component development. Nanotubes with exceptionally stiff and strong mechanical properties can help us make lightweight structural components. Nanotubes as capsules can help us store and carry hydrogen and other hydrocarbon-based fuel in automobiles or aboard spacecraft. Carbon-based materials are ideally suitable as molecular-level building blocks for nanoscale system design, fabrication, and applications. From a structural or functional materials perspective, carbon is the only element that exists in a variety of shapes and forms with varying physical and chemical properties. All basic shapes and forms needed to build any complex molecular-scale architecture are already available with carbon.

\section{Kinetics of Carbon Nanotubes}

Carbon nanotubes can be produced small enough to pass through holes in tumors or to transport DNA, because their high surface ratio to volume ratio provides a good platform for efficient transportation of chemicals and for the reactions needed for ultrasensitive glucose detection [2,3]. Their very small size and their ability to contain chemicals also contribute to their properties as drug carriers and/or drug delivery, which are very relevant in cancer research and therapy. As drug carriers, the administration, absorption, and transportation of CNTs must be considered for obtaining the desired treatment effects. The studied routes of CNT administration include oral and injections such as subcutaneous injection, abdominal injection, and intravenous injection. There are different ways of absorption and transportation when CNT's are administered by different routes. The absorbed CNT's are transported from the administration sites to the effect-relevant sites by blood or lymphatic circulation. After administration, absorption is the first key step for drug carriers to complete their drug- delivering mission.

Studies have suggested that CNTs themselves are capable of being absorbed. It has also been established that physically shortened CNTs that are orally administered can be absorbed through the columnar cells of intestinal mucous membrane, where this was confirmed by transmission electron microscopy $[4,5]$. Distribution indicates the sites or places the absorbed CNTs can arrive and exist, of great importance in clinical pharmacology and toxicology of CNTs as drug carriers. There have been experiments to investigate in vivo and ex vivo biodistributions, as well as tumor targeting ability of radiolabeled SWCNTs (diameter, approximately 1 to $5 \mathrm{~nm}$, length, approximately 100 to $300 \mathrm{~nm}$ ) noncovalently 
functionalizes with phospholipids (PL)-PEG in mice using positron emission tomography and Raman spectroscopy, respectively. It was interesting to note that the PEG chain lengths determine the biodistribution and circulation of CNTs. Note that PEG stands for polyethylene glycol.

The nonbiodegradability in the body and non-eliminability from the body raise questions on the possibility of their successful use in clinical practice, factors which have always been a concern. Functionalized SWCNTs seem to be metabolizable in the animal body. For example, SWCNTs with carboxylate surfaces have demonstrated their unique ability to undergo 90-day degradation in a phagolysosome simulant, resulting in shortening of length and accumulation of ultrafine solid carbonaceous debris. Unmodified, ozonized, aryl sulfonated SWCNTs exhibit no degradation under similar conditions. The observed metabolism phenomenon may be accredited to the unique chemistry of acid carboxylation, which, in addition to introduction the reactive, modifiable $\mathrm{COOH}$ groups onto CNT surfaces, also induces collateral damage to the tubular graphemic backbone in the form of neighboring active sites that provide points of attack for further oxidative degradation $[6,7]$.

\section{Opening Filling and Capping CNTs}

As mentioned previously, carbon nanotubes are end capped and thus for drug loading there are essentially two approaches which include the filling of carbon nanotubes during synthesis or after synthesis. Adding the contents of the nanotubes in-situ tends to be a less efficient approach, producing a yield of around 10\% whereas the post-synthesis process can be better controlled and yields of $\sim 50 \%$ on the material that is to be inserted into the CNT. The criteria include melting temperature, reactiveness, surface tension and sensitivity of the material. Post-synthesis production of CNTs implies that the ends must be opened. This can be accomplished by passing electric currents through the CNT, through attacking the CNT with acid which corrodes the angled parts of the tube the most (i.e. the ends), or by oxidization using carbon dioxide [8-10]. There are two ways to include foreign particles in CNTs. One category is decoration, which is the process of bonding a functional group to CNTs $[11,12]$.

This is difficult as carbon is rather inert, so oxidization is used to produce a more reactive attachment surface. The functional group is either bonded to the inside or outside of the walls. The most common mechanism for filling CNTs is capillarity. The limiting factor in capillarity is the diameter of the CNT and the surface tension of the material (the threshold material surface tension is approximately $200 \mathrm{mN} / \mathrm{m}$ ). However, hydrophobic and Van der Waals forces also play a role in aqueous solutions. For chemicals with higher surface tensions it is possible to lower this tension by creating a suitable composite, which can be chemically reduced to the original substance once the CNT has been filled. The CNTs are washed using a solution which has been chosen to offer only limited solubility to the impregnating fluid and thus can dissolve only deposits left outside the CNT. After filling, the CNTs are capped by passing a current which fuses the ends closed $[13,14]$. The loading of CNTs remains an area requiring further research and more frequently mathematical methods are used rather than laboratory experiments due to the comparatively lower cost $[15,16]$.

\section{CNTs Functionalization}

Functionalization helps in making CNTs more soluble than the impurities by attaching other groups to the tubes and this will make it easy to separate from insoluble impurities, such as metal, this is usually done using filtration [17-19]. Functionalization technique also leaves the CNT structure intact and makes them soluble for chromatographic size separation. For recovery of the purified CNTs, the functional groups can be simply removed by thermal treatment, such as annealing [20-23].

\section{Targeting Methods}

Previous attempts at antibody-mediated drug delivery have been largely unsuccessful due to the loss of specificity of the antibodies on binding with drug molecules. It was found that using nanotubes to support antibodies did not change their properties and so did not inhibit their targeting abilities. Targeting methods such as active or passive targeting are a direct result of functionalization. Passive targeting is a result of inertness and physical size of the macromolecule, "hiding" it from the immune system. CNTs must be nanosized to prevent cellular opsonization (the susceptibility of the macromolecule to ingestion by phagocytes resulting in its destruction) by the innate immune system but also functionalized with molecules/polymer chains such as PEG which do not promote an adaptive immune response. The CNT must also be of sufficient size to utilize the EPR effects and so a trade-off is required. PEG is useful in determining the degree of optimal functionalization as it is an easily controllable variable. This passive targeting can cause problems; microspheres can lead to chemo embolism-type problems in the lymphatic nodes.

For such cases, functionalization with nanomagnetic particles (e.g. iron oxide) and placing of a magnet at the desired location for extended periods of time allows for drug release over an extended period. Active targeting requires functionalization with tumorspecific binding sites to selectively bind to tumor cells. Many cells of various cancers are known to over press certain receptors such as brain tumors receptors [24-26].

\section{Applications of CNTs as Drug Carriers}

\section{Anticancer Molecules}

It is well known that cancer cells overexpress folic acid (FA) receptors, and several research groups have designed nanocarriers with engineered surfaces to which FA derivates can be attached. Moreover, no spherical nanocarriers (e.g., CNTs) have been reported to be retained in the lymph nodes for longer periods of time compared to spherical nanocarriers [27] (e.g., liposomes). Thus, 
CNTs might be used for targeting lymph node cancers as shown by various investigators. In these studies, magnetic nanoparticles containing the anticancer cisplatin were entrapped into folic-acidfunctionalized MWNTs. An external magnet was employed to drag the nanotubes to the lymph nodes where the drug was shown to be released over several days and the tumor to be selectively inhibited. In a recent study [28] loaded the anticancer molecule gemcitabine into magnetic MWNTs and, using mice, they reported high activity against lymph node metastasis when the formulation was injected subcutaneously [28]. In another study, the poorly water-soluble anticancer calprotectin has been loaded into polyvinyl alcohol functionalized MWNTs and reported to be potentially effective in treatment of breast and skin cancers [29].

\section{Lymphatic System}

Many cancers metastasize through the lymphatic canal. Drug delivery systems targeted to the lymphatic system can block the metastasis of cancers effectively. Using radical polymerization polyacrylic acid (PAA) can be extended onto CNTs, making them highly hydrophilic. Through coprecipitation, $\mathrm{Fe}_{3} \mathrm{O}_{4}$ based magnetic nanoparticles can be adsorbed on the PAA-CNT surface. Through the interaction with $\mathrm{COOH}$ groups of grafted PAA, the nanoparticles can be stabilized from clustering. By stirring the solution containing PAA-CNT, Fe304 -based magnetic nanoparticles, and gemcitabine for $24 \mathrm{~h}$, gemcitabine was loaded into the nano system with a loading efficiency of $62 \%$. It was found that CNTs were seen only in the local lymphatic nodes and were absent in the major organs, such as liver, kidney, heart, spleen, and lungs, after $3 \mathrm{~h}$ of subcutaneous injection. Without the help in addition to covalent attachment, anticancer drugs can also attach to the surface of the CNT by noncovalent bonding.

This involves physical conjugation of the drug to CNT via $\pi-\pi$ stacking, hydrophobic interaction, or electrostatic adsorption. Although covalent attachment is a very feasible procedure, it has been suggested that this may cause chemical changes in anticancer drugs, implying that their efficacy can potentially be altered [30]. However, one of the disadvantages of noncovalent bonding is the lack of efficient attachment, potentially resulting in release of the drug before it reaches its site of action [31,32]. An example of noncovalent attachment of an anticancer drug in this context is the attachment of doxorubicin to MWCNTs. In one experiment, MWCNTs were dispersed in 1\% Pluronic $®$ F127 solution until a final MWCNT concentration of $1 \mathrm{mg} / \mathrm{mL}$ was formed. The solution was then bath-sonicated for 30 minutes. Increasing concentrations of Pluronic-MWCNTs $(10,20$, and $40 \mu \mathrm{g} / \mathrm{mL})$ were then reacted with doxorubicin $20 \mu \mathrm{g} / \mathrm{mL}$.

The interaction between the MWCNTs and doxorubicin was studied using luminescence spectrometry. The results showed that the fluorescence intensity of doxorubicin decreased with increasing concentrations of MWCNT. This suggests that as the concentration of the MWCNT increases, more platforms become available for noncovalent interaction of doxorubicin with the surface of the MWCNT. In another experiment, pegylated CNTs were reacted with doxorubicin, resulting in doxorubicin becoming loaded onto the PEG that was covering the surfaces of the CNTs. It was suggested that, due to the aromatic nature of doxorubicin, noncovalent binding of this molecule onto the surface of the CNT was most likely because of $\pi-\pi$ stacking and hydrophobic interactions [33].

\section{Blood-Brain Barrier}

The location of the drug to be delivered by the CNT can be internal or external. Internalization or encapsulation relies on Van der Waals forces for insertion into the CNT and is best used for drugs that are sensitive to external environments and easily broken down [34]. In the case of drug transport at the blood-brain barrier, $\mathrm{BBB}$, the physiological function of the BBB is to maintain brain homeostasis by selectively transporting nutrients and beneficial endogenous substances into the brain and excluding toxic metabolic or xenobiotics from the brain. The pivotal component of the BBB is a monolayer of brain capillary endothelial cells fused by tight junctions. Other components of the BBB including the astrocytic foot process, pericytes and perivascular macrophages within a basal lamina regulate and further strengthen the BBB $[35,36]$. In addition to tight junctions, the absence of fenestrations also contributes to the barrier property of brain endothelial cells.

Furthermore, in contrast to vascular endothelial cells in other tissues, the low activity of pinocytosis and vesicular traffic further limits non-specific trans-endothelial transport with the exception of small lipid-soluble molecules [37]. Transport mechanisms at the BBB can be divided into two categories: passive diffusion and endogenous carrier-mediated transport. Passive diffusion is a process whereby drugs or endogenous substances travel across the BBB dependent upon along a concentration gradient from blood to brain, and the physicochemical properties of the drug. Qualitatively, drugs that passively diffuse through the BBB are generally lipophilic, often related to the octanol/water partition coefficient, and have a molecular weight of less than 400-500 Da. Numerous quantitative relationships have been cast to correlate BBB penetration to lipophilicity and molecular weight as well as other chemical structural features [37-39].

\section{Concluding Remarks}

Much of the recent progress in modern nanotechnology is in the experimental biomolecular motor's arena. Researchers have gained significant understanding about how the natural biological motor systems work and how to create interfaces of nanoscale biomolecular motors with synthetic materials in solution-phase environments [40]. Means to power these machines through biomimetic physical and chemical phenomenon are also under investigation. Ultimately, we might see nanoscale synthetic machines and motors that are powered and controlled through external laser, electric, or magnetic fields and that could operate 
in chemical solution phases or inert gas environments [41,42]. A final issue concerns building the world atom-by-atom from the bottom up-the holy grail of molecular technology. Experimentally, certain areas could contribute significantly toward advances in this direction. These areas are nanomanipulation and control of atomicand-molecular-level entities on solid, 3D surfaces and self-assembly of molecular-scale materials where chemical and topological structural forces can guide chunks of nanoscale materials toward larger functional materials structures. Advanced nanotechnology can and does contribute significantly to this by simulating the assembly of building-block materials.

One such contribution is concerned with the review reported here on carbon nanotubes and their application to carbon research and therapy, which depends enormously of the unique advantages of CNTs. In addition to their properties such as large aspect ratio and ability to pass through cellular membranes and so on the reason why these nanoparticles are marked out as distinctive is because they are compatible with the whole notion of multiple functionalization, which other delivery systems benefit very little from. In other words, CNTs could be decorated with various compounds each of which exhibits a different role: targeting agents to reduce side effects, drugs to provide therapeutic effects, stealth agents to avoid the immune system, and diagnostic agents to monitor the location of delivery system. In concert with the direction that drug delivery is headed, this notion is certainly of paramount significance as the drug delivery systems are ultimately sought to accomplish far more than spraying therapeutic agents to tissues.

An exceptional as they look to be, the (bio)chemistry of CNTs raise some serious concern, as well. The fundamental problem we face is their nonbiodegradable quality. Unlike liposomes, they are not disposable and hence the biological fate of drug delivery systems after releasing drugs in cells remains a little ambiguous. Also, there is no consensus on their toxic effects, which may vary depending on the study and the material utilized. (vide supra). Hence, extensive studies on their toxicology profile and their synthesis is a must for us to fully comprehend how these materials behave in biological media. When viewed from this perspective, these disadvantages appear to outweigh all the previously mentioned profits and hold up the commercialization of CNTs-based systems. In short, what we accomplished so far with these nanostructures is a huge step forward, considering what is summarized in this article. However, there is still a long way to go before making some tailored drugs delivery systems out of CNTs.

\section{Acknowledgement}

Note: I would like to thank Liliana Carvalho for manuscript editing.

This research did not receive any specific grant from funding agencies in the public, commercial, or not-for-profit sectors.

\section{References}

1. Dressel Haus MS, Dressel Haus D, Eklund PC (1996) Science of Fullerenes and Carbon Nanotubes, Academic Press, New York.

2. Chang CH, Lin WJ, Liao YM, Chow HY, Chen YF, et al. (2018) Selfpowered ultrasensitive glucose detection based on graphene multiheterojunctions. Conference on Lasers and Electro-optics OSA Technical Digest paper AM4P4.

3. Mohammadi S, Taheri A, Rezayati Zad Z (2018) Ultrasensitive and selective non-enzymatic glucose detection based on $\mathrm{Pt}$ electrode modified by carbon nanotubes @graphene oxide/nickel hydroxideNafion hybrid composite in alkaline media. Prog Chem Biochem Res 1: $1-10$.

4. Son KH, Hong JH, Lee JW (2016) Carbon nanotubes as cancer therapeutic carriers and mediators. Int J Nanomedicine 11: 5163-5185.

5. Ali Boucetta H, Kostarelos K, Al Jamal KT, Mc Carthy D, Prato M, et al. (2008) Multiwalled carbon nanotube-doxorubicin supramolecular complexes for cancer therapeutics. Chem Commun (Camb) 4: 459-461.

6. Liu X, Tao H, Yang K, Zhang S, Lee ST, et al. (2011) Optimization of surface chemistry on single-walled carbon nanotubes for in vivo photothermal ablation of tumors. Biomaterials 32: 144-151.

7. Pujiastuti S, Wulan PPDK (2019) The effect of hydrogen peroxide on carbon nanotubes solubility as drug delivery material for cancer with covalent functionalization. AIP Conference Proceedings 2092(1): 030006 .

8. Ebbesen TW (1996) Wetting filling and decorating carbon nanotubes. J Phys Chem Solids 57: 951-955.

9. Ajayan PM, Ebbesen TW, Ichihashi T, Iijima S, Tanigaki K, et al. (1993) Opening carbon nanotubes with oxygen and implications for filling. Nature 362: 522-525.

10. Sugimoto T (Eds.)., (2000) Fine Particles. Synthesis, Characterization, and Mechanisms of Growth. Marcel Dekker, Inc. New York, USA.

11. Gao YK, Dx C, Cs O (2003) Spontaneous insertion of DNA oligonucleotides into carbon nanotubes. Nano Letters 3(4): 471-473.

12. Yang J, Fujigaya T, Nakashima N (2017) Decorating unoxidized carbon nanotubes with homogeneous Ni-Co spinel nanocrystals show superior performance for oxygen evolution/reduction reactions. Sci Rep 7: 45384.

13. Dejonge N, Doytcheva M, Allioux M, Kaiser M, Mentink S, et al. (2005) Cap closing of thin carbon nanotubes. Adv Mater 17(4): 451-455.

14. Ono S, Tanikawa K, Kuwahara R, Ohno K (2016) Relationship between cap structure and energy gap in capped carbon nanotubes. J Chem Phys 145(2): 024702.

15. Heister E, Neves V, Tolmaciu C, Lipert K, Sanz Beltrón V, et al. (2009) Triple functionalization of single-walled carbon nanotubes with doxorubicin, a monoclonal antibody, and a fluorescent marker for targeted cancer therapy. Carbon 47: 2152-2160.

16. Yas MH, Heshmati M (2012) Dynamic analysis of functionally graded nanocomposite beams reinforced by randomly oriented carbon nanotube under the action of moving load. Appl Mathematical Modelling 36: 1371-1394.

17. Mallakpaur S, Soltanian S (2016) Surface functionalization of carbon nanotubes fabrication and applications. RSC Advances 6(111): 109916109935.

18. Georgakilas V, Kordatos K, Prato M, Guidi DM, Holzinger M, et al. (2002) Organic functionalization of carbon nanotubes. J Am Chem Soc 124: 760761.

19. Chen RJ, Bengsaruntij S, Drouvalakis KA, Kam NWS, Shim M, et al. (2003) Noncovalent functionalization of carbon nanotubes highly specific electronic biosensors. Proc Natl Acad Sci USA 100(9): 4984-4989. 
20. Monthioux M (2002) Filling single-wall carbon nanotubes. Carbon 40(10): 1809-1823.

21. Vardharajula S, Ali SZ, Tiwari PM, Eroglu E, Vig K, et al. (2012) Functionalized carbon nanotubes: biomedical applications. Int J Nanomedicine 7: 5361-5374.

22. Zhou Y, Fang Y, Ramasamy RP (2019) Non-covalent functionalization of carbon nanotubes for electrochemical biosensor development. Sensors 19(2): 392-420.

23. Pérez Luna V, Moreno Aguilar C, Arauz Lara JL, Aranda Espinoza S, Quintana M, et al. (2018) Interactions of functionalized multi-wall carbon nanotubes with giant phospholipid vesicles as model cellular membrane system. Sci Rep 8: 17998.

24. Zwicke GL, Ali Mansoori G, Jeffery CJ (2012) Utilizing the folate receptor for active targeting of cancer nanotherapeutics. Nano Reviews 3: 18496

25. Kumari P, Ghosh B, Biswas S (2016) Nanocarriers for cancer-targeted drug delivery. J Drug Target 24(3): 179-191.

26. Attia MF, Anton N, Wallyn J, Omran Z, Vandamme T, et al. (2019) An overview of active and passive targeting strategies to improve the nanocarriers efficiency to tumour sites. J Pharmacy and Pharmacology 71: 1185- 1198.

27. Liu Z, Chen K, Davis C (2008) Drug delivery with carbon nanotubes for in vivo cancer treatment. Cancer Research 68(16): 6652-6660.

28. Cheng J, Meziani MJ, Sun YP, Cheng SH (2011) Poly(ethylene glycol) - conjugated multi-walled carbon nanotubes as an efficient drug carrier for overcoming multidrug resistance. Toxicology and Applied Pharmacology 250(2): 184-193.

29. Liu X, Tao H, Yang K, Zhang S, Lee ST, et al. (2011) Optimization of surface chemistry on single-walled carbon nanotubes for in vivo photothermal ablation of tumors. Biomaterials 32: 144-151.

30. Yang Z, Zhang Y, Yang Y, Sun L, Han D, et al. (2010) Pharmacological and toxicological target organelles and safe use of single-walled carbon nanotubes as drug carriers in treating Alzheimer disease. Nanomedicine 6(3): 427-441.

ISSN: 2574-1241

DOI: $10.26717 / B J S T R .2020 .25 .004252$

César A C Sequeira. Biomed J Sci \& Tech Res

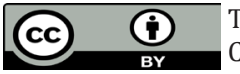

This work is licensed under Creative Commons Attribution 4.0 License

Submission Link: https://biomedres.us/submit-manuscript.php
31. Liu Z, Sun X, Nakayma Ratchford N, Dai H (2007) Supramolecular chemistry on water-soluble carbon nanotubes for drug loading and delivery. ACS Nano 1(1): 50-56.

32. Rastogi V, Yadav P, Bhattacharya SS, Mishra AK, Verma N, et al. (2014) Carbon nanotubes: An emerging dug carrier for targeting cancer cells. J Drug Deliv pp. 670815.

33. Jiang ZS, Sun YZ, Wang SM, Ruan JS (2017) Epithelial-mesenchymal transition: potential regulator of $\mathrm{ABC}$ transporters in tumor progression. J Cancer 8(12): 2319-2327.

34. Hillebrenner H, Buyukserin F, Kang M, Mota MO, Stewart JD, et al. (2006) Corking nano test tubes by chemical self-assembly. J Am Chem Soc 128(13): 4236-4237.

35. Abbot NJ, Ronnback L, Hansson E (2006) Astrocyte endothelial interactions at the blood brain barrier. Nat Rev Neurosci 7(1): 41-53.

36. Campisi M, Shin Y, Osaki T, Hajal C, Chiono V, et al. (2018) 3D-self organized microvascular model of the human blood-brain barrier with endothelial cells, pericytes and astrocytes. Biomaterials 180: 117-129.

37. Caplan LR, Biller J, Leary MC, Lo EH, Thomas AJ, et al. (2017) Primer on cerebrovascular Diseases, $\left(2^{\text {nd }} E d n\right.$.). Academic Press London.

38. Greenberg HS, Chandler WF, Sandler HM (1999) Brain Tumors. Oxford University Press New York

39. Levin VA, Ellingson BM (2018) Understanding brain penetrance of anticancer drugs. Neuro-Oncology 20(5): 589-596.

40. Atakan B (2014) Molecular Communication and Nanonetworks, From Nature to Practical Systems. Springer New York, USA.

41. Schuz MJ, Shanov VN, Yin Z (Eds.)., (2014) Nanotube Super fiber Materials. Changing Engineering Design. Elsevier Amsterdam.

42. Li S, Gao XL (Eds.)., (2013) Handbook of Micromechanics and Nano mechanics. CRC Press. Taylor and Francis Group Boca Raton FL.

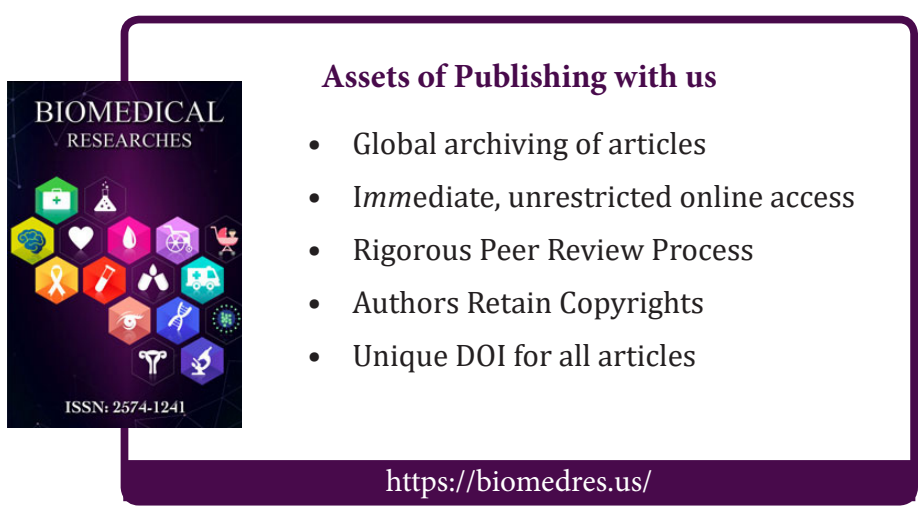

